Portland State University

PDXScholar

Electrical and Computer Engineering Faculty

Publications and Presentations

5-1-1989

\title{
Direct Current-Excited CW CO² Metal Waveguide Laser
}

\author{
Fahad S. Al-Mashaabi \\ Portland State University \\ Lee W. Casperson \\ Portland State University
}

Follow this and additional works at: https://pdxscholar.library.pdx.edu/ece_fac

Part of the Electrical and Computer Engineering Commons

Let us know how access to this document benefits you.

\section{Citation Details}

Fahad S. Al-Mashaabi and Lee W. Casperson, "Direct current-excited cw CO2 metal waveguide laser," Appl. Opt. 28, 1897-1903 (1989).

This Article is brought to you for free and open access. It has been accepted for inclusion in Electrical and Computer Engineering Faculty Publications and Presentations by an authorized administrator of PDXScholar. Please contact us if we can make this document more accessible: pdxscholar@pdx.edu. 


\title{
Direct current-excited $\mathrm{cw} \mathrm{CO}_{2}$ metal waveguide laser
}

\author{
Fahad S. Al-Mashaabi and Lee W. Casperson
}

\begin{abstract}
A novel design for a dc-excited $\mathrm{cw} \mathrm{CO} \mathrm{CO}_{2}$ metal waveguide laser has been developed in which a slotted hollow cathode also doubles as a metal waveguide for the cavity modes. This design has been implemented in a compact structure that produces over $1 \mathrm{~W}$ of $\mathrm{cw} 10.6-\mu \mathrm{m}$ radiation. The discharge characteristics, laser gain, and laser output have been studied as functions of various discharge parameters. The advantages of the transverse discharge of the slotted hollow cathode geometry include low voltage, positive impedance, rugged structure, and high optical gain. Overall efficiency is comparable with that of conventional longitudinal $\mathrm{CO}_{2}$ lasers. The output laser modes are clean low-order Hermite-Gaussian or Airy function modes.
\end{abstract}

\section{Introduction}

Since the demonstration of the first $\mathrm{CO}_{2}$ waveguide laser by Smith ${ }^{1}$ in 1971 , in which the walls of the laser structure serve as an enclosure to contain the gas mixture and as a waveguide to confine the laser light, various waveguide designs and plasma excitation methods have been investigated to meet the demand for a compact, rugged, and efficient laser. ${ }^{2,3}$ Waveguides used for guiding IR radiation are either nonflexible or flexible. Nonflexible waveguides are usually used to guide the electromagnetic radiation inside the laser cavity. The modes of these waveguides have been studied for hollow dielectric waveguides with circular cross sections ${ }^{4}$ and rectangular cross sections ${ }^{5}$ and for composite metal and dielectric waveguides of rectangular cross sections. ${ }^{6}$ Flexible waveguides that are usually used to deliver $\mathrm{CO}_{2}$ laser beams to targets are either flexible metal ${ }^{7,8}$ or IR fiber optic ${ }^{9,10}$ waveguides. There are often clear advantages to using fiber optic waveguides for low power applications, but for high power applications metallic waveguides have proved to be the best choice.

To couple efficiently a laser beam into a waveguide, Marhic et al. ${ }^{11}$ proposed a laser whose output modes match the waveguide modes. This is done so that the walls of the laser active medium are made up of a portion of the waveguide itself or have a similar shape to it. In this work we are interested mainly in metallic

The authors are with Portland State University, Department of Electrical Engineering, Portland, Oregon 97207-0751.

Received 21 October 1988.

0003-6935/89/101897-07\$02.00/0.

(C) 1989 Optical Society of America. waveguides. A metallic waveguide could be a hollow tube $^{7}$ or a concave strip. ${ }^{8}$ Resonators of this type have been experimentally studied, and examples are the $\mathrm{cw}$ $\mathrm{CO}_{2}$ laser with a slightly bent glass tube ${ }^{11}$ and the transversely rf-excited metal waveguide laser ${ }^{12}$ with a concave metal strip waveguide. Although the output modes of both lasers have been shown to agree well with the expected Airy-Hermite-Gaussian functions, ${ }^{8}$ there are practical differences between the two schemes. The glass tube laser suffers from very inefficient use of the gain medium. The second laser is more efficient but is more expensive because it requires a rf power supply and impedance matching to the laser discharge. Another problem is the potential for rf electromagnetic interference. These problems may be simultaneously eliminated by designing a new type of waveguide discharge that is able to generate a relatively high gain medium using dc excitation.

We report the results of a detailed study of a new design for metallic waveguide lasers using transverse dc excitation. In this design the cathode, which also serves as a waveguide, is a slotted hollow tube. This new type of waveguide laser has been constructed and investigated. The compact size, rugged structure, positive discharge impedance, and good modal quality make this metal waveguide laser suitable for many applications, such as laser surgery, laser machining, and laser radar. The design and discharge characteristics of this waveguide laser device are described in Sec. II.

The gain measurements, which are a necessary step in assessing the suitability of our new waveguide discharge for laser excitation and in obtaining the optimum discharge conditions for laser action, are presented in Sec. III. With the addition of an optical resonator the amplifier becomes a laser oscillator. In Sec. IV, the operation of this laser is studied by observing the transverse output modes and measuring the 
output power as functions of various discharge parameters.

\section{Slotted Hollow-Cathode Discharge}

A slotted hollow metal tube has been developed which serves as both a waveguide and the cathode in a transverse discharge. This new waveguide discharge has many of the characteristics of both plane-cathode and hollow-cathode discharges. The discharge voltage is low being of the order of $370-450 \mathrm{~V}$. Also, the discharge exhibits a positive impedance. As a result, the overall efficiency is increased, since very small or zero ballast resistance can be used. The optical gain measurements reveal that a substantial gain is available, and lasing has been achieved. This metal waveguide laser consists (as shown in Fig. 1) of three main parts - the cathode (waveguide), anode, and resonator structure.

The cathode material and shape is a simple copper tube (i.d. $=1.1 \mathrm{~cm}$ ) which is slotted along its center axis with a slot width of $0.9 \mathrm{~cm}$. The cathode is also bent manually along the optical axis to have the desired radius of curvature. Copper is chosen for its excellent $10.6-\mu \mathrm{m}$ optical properties, low work function, and low susceptibility to sputtering. ${ }^{13}$ The polished inner surface of the cathode also retains its surface cleanliness during laser operation, thus eliminating the need for the toxic and expensive berylium oxide or other materials such as aluminum oxide and boron nitride, which are sometimes used as waveguide walls. Cooling of the copper cathode in our design is provided by tap water that flows through a small diameter copper pipe (i.d. = $0.5 \mathrm{~cm}$ ) attached to the outer surface of the cathode.

The anode material of this device is not critical, and no noticeable differences in the V-I characteristics were observed when different metals were tested. The anode principally used during our experiments was a 3$\mathrm{mm}$ diam brass rod. The anode is supported by another three rods through hinged connections that allow the matching of the anode's radius to the major radius of curvature of the cathode. The resonator structure is enclosed in a vacuum tight Plexiglass tube [i.d. = $8.89 \mathrm{~cm}$ (3.5 in.), o.d. $=10 \mathrm{~cm}(4 \mathrm{in})$.$] that is O-ring$ sealed at both ends with Plexiglass plates. These plates also hold the two adjustable mirror mounts.

The device is connected to a power supply of $(1.0 \mathrm{kV}$, $1.5 \mathrm{~A})$ to sustain the discharge. Although the discharge has a positive impedance, a low $1-\mathrm{k} \Omega$ ballast resistor is sometimes used to stabilize the discharge and to protect the power supply from sudden arcs that could be caused by defects on the cathode surface. The ratios of the input gas mixture of $\mathrm{CO}_{2}: \mathrm{N}_{2}: \mathrm{He}$ are controlled by three Dwyer flow meters. The flow rate of the gas mixture has been kept constant at $\sim 1$ SCFH (standard cubic foot per hour).

A hollow-cathode discharge is sometimes used to form a strong negative glow by the superposition of two negative glows opposite to each other. Usually the hollow cathode is cylindrical in shape. The qualitative theory of hollow-cathode discharge design including such effects as cathode-cathode separation and

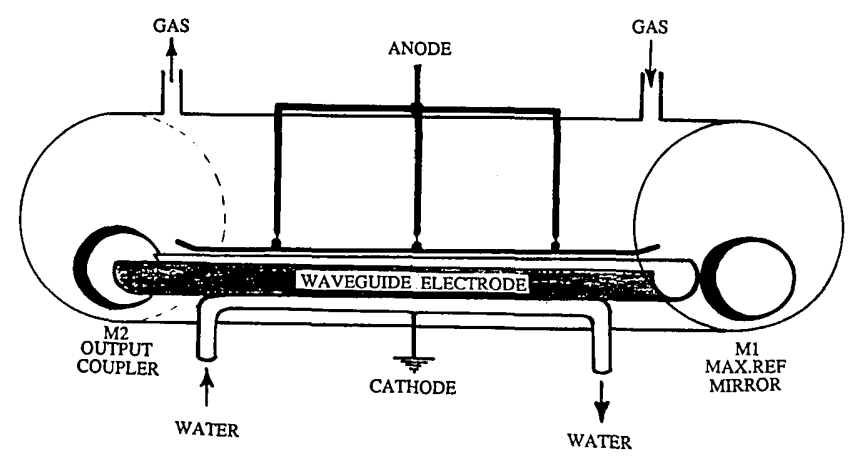

Fig. 1. Directcurrent-excited $\mathrm{CO}_{2}$ metal waveguide laser.

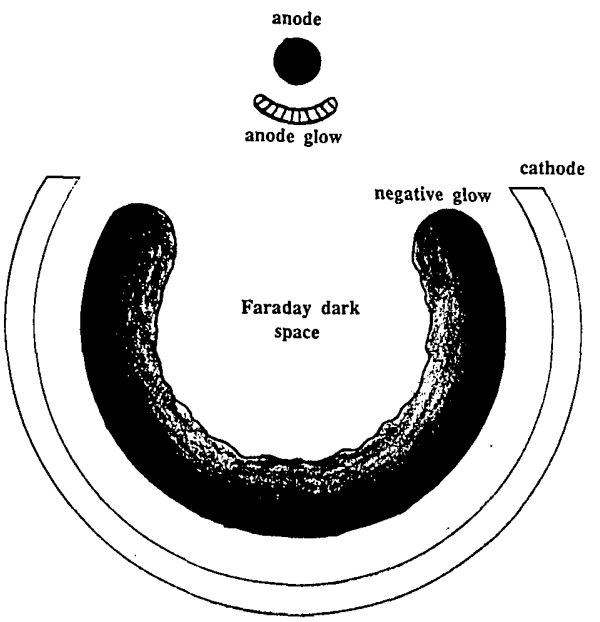

Fig. 2. Slotted hollow cathode discharge system configuration.

anode-cathode separation is presented in Ref. 14 . More quantitative discussions are presented in Refs. 15-17. There are two basic groups of hollow-cathode discharge designs, transverse and longitudinal, depending on the direction of the electric field with respect to the cathode axis. Various types of hollowcathode discharge have been used as gain media for $\mathrm{He}-\mathrm{Ne}$ lasers, ${ }^{18} \mathrm{He}-\mathrm{Cd}$ lasers, ${ }^{19}$ argon-ion lasers, and other high lying ionic level lasers.

A single pass gain of $10 \% / \mathrm{m}$ has been reported in the negative glow of a hollow-cathode discharge in a $\mathrm{CO}_{2}-$ He mixture. ${ }^{20}$ In our attempt to obtain higher gain and also to get a more flexible cathode (waveguide), we introduced a slot along the cathode axis. Figure 2 illustrates the cross section of this discharge. The advantages of this configuration can be summarized as follows: (1) rigid and high thermal conductivity metal cavity; (2) low discharge voltage; (3) positive impedance similar to the abnormal glow of a plane cathode discharge; and (4) homogeneity of the discharge along the cathode center (optical axis).

In our work, we used the Faraday dark space formed in the middle of the cathode cross section as the laser gain medium, and there are three reasons for this choice: First, the negative glow is not a practical plasma for the excitation of the upper $\mathrm{CO}_{2}$ laser level. ${ }^{20}$ Second, the positive column is hard to obtain inside the hollow cathode. Third, gain has been reported 
previously by Freiberg and Clark ${ }^{21}$ in the Faraday dark space. Obtaining the Faraday dark space in the middle of the cathode cross section is done by adjusting the gas pressure, mixture ratio of $\mathrm{CO}_{2}: \mathrm{N}_{2}: \mathrm{He}$, and current and by using a relatively large diameter (in our case i.d. $=1.1 \mathrm{~cm}$ ). As a result, the negative glow forms a $\mathrm{C}$ shape close to the cathode surface with a thickness of $\sim 2 \mathrm{~mm}$, and this thickness changes depending on the discharge parameters. From visual observation, the anode glow was always found to exist at the attainable pressure range (6-15 Torr), while no positive column was observed. The Faraday dark space is somewhat unusual because of the focusing effect of the slotted hollow cathode configuration compared to a plane cathode geometry. In this Faraday dark space, the $\mathrm{CO}_{2}$ laser gain was found to be comparable to the gain in the regular longitudinal positive column plasma for the same gas pressure, mixture ratio, and flow rate. Figure 3 shows the V-I characteristics exhibited by the transverse hollow-cathode discharge for different gas mixtures.

\section{Gain Measurements}

Single pass gain measurements have been performed as a function of gas mixture ratio, pressure, current, and radial distance from the cathode. Other measurements such as gain as a function of $E / P$ and gas temperature were not performed. Since the operating pressures are all above 5.2 Torr, the effect of Doppler broadening is small compared to collisional broadening. ${ }^{22,23}$ Therefore, in deriving the small signal gain equation, the inhomogeneous line broadening is ignored. The homogeneous linewidth is $\sim 100 \mathrm{MHz}{ }^{24}$

For a gain medium of length $L$ the gain is given by

$$
\begin{aligned}
g_{0} & =\frac{1}{L} \ln \frac{I(L)}{I(0)} \\
& =\frac{1}{L} \ln \frac{I_{\text {on }}}{I_{\text {off }}},
\end{aligned}
$$

where $I_{\text {on }}=$ intensity with discharge on and $I_{\text {off }}=$ intensity with discharge off.

The gain measurements were performed using the apparatus illustrated in Fig. 4. A conventional low power $\mathrm{CO}_{2}$ laser was used to provide a low order TEM mode probe beam. The higher-order modes were eliminated by an aperture. The probe beam laser has a $1-\mathrm{cm}$ diam $150-\mathrm{cm}$ long water cooled discharge tube with a Brewster window at each end. The discharge tube was placed between a $2-\mathrm{m}$ gold coated curved mirror and a flat germanium mirror with $80 \%$ reflectivity, forming a $185-\mathrm{cm}$ long resonator. The discharge current was $15-25 \mathrm{~mA}$ at $5-8 \mathrm{kV}$. The gas mixture $\mathrm{CO}_{2}: \mathrm{N}_{2}: \mathrm{He}$ of $1: 1: 8$ was used at a pressure of $7-10$ Torr.

Since the probe beam is invisible, a He-Ne laser was used to show the probe beam path, as shown in Fig. 4. The flat mirrors $M_{1}$ and $M_{2}$ provided the alignment of the He-Ne laser. The available beam splitter $M_{3}(80 \%$ reflectivity at $10.6 \mu \mathrm{m}$ ) served as the point where the invisible $10.6-\mu \mathrm{m}$ beam merged with the $\mathrm{He}-\mathrm{Ne}$ beam. After this point, the two beams propagate collinearly, and $M_{4}$ (maximum reflectivity at $10.6 \mu \mathrm{m}$ ) redirects

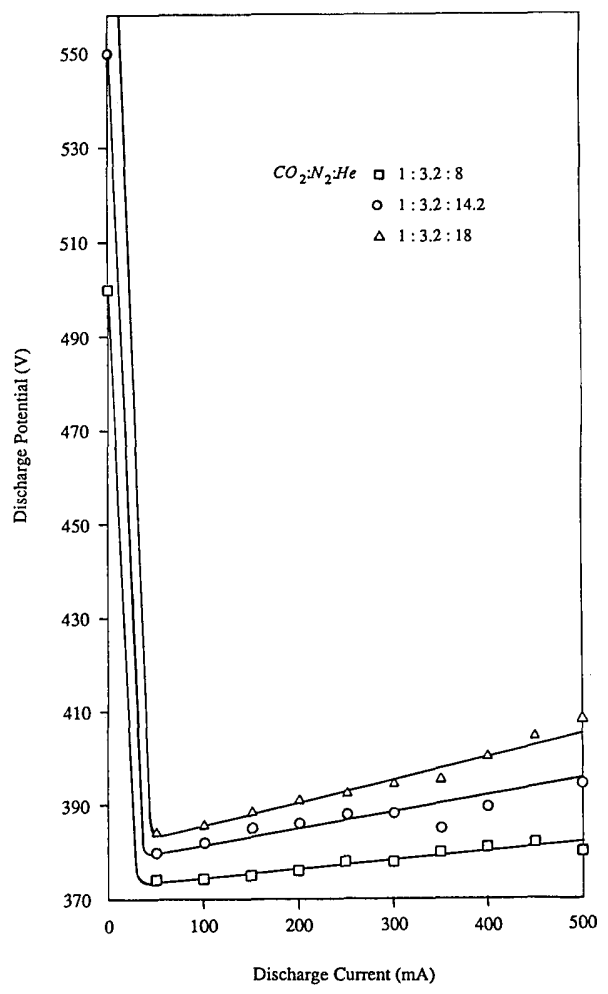

Fig. 3. Voltage-current characteristics of the waveguide discharge.

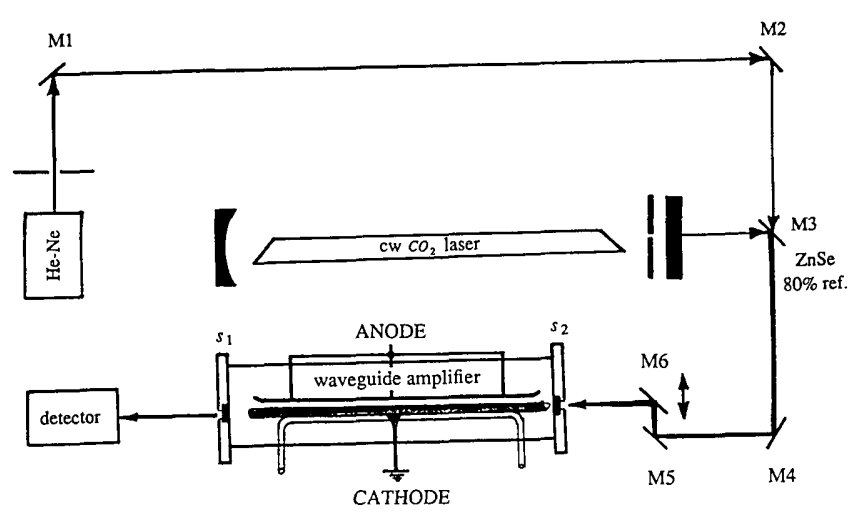

Fig. 4. Gain measurement apparatus.

the beam to $M_{5}$. The two mirrors $M_{5}$ and $M_{6}\left(M_{6}\right.$ is maximum reflectivity at $10.6 \mu \mathrm{m}$ ) provide the alignment of the two beams to propagate through the waveguide parallel to its axis.

The probe beam is scanned across the amplifier cross section by translational movement of $M_{6}$. The salt windows $s_{1}$ and $s_{2}$ are mounted at the ends of the amplifier. After passing through the amplifier, the probe beam is detected by a Scientech 362 power meter. Equation (1) requires two sets of power measurements when the discharge is on and off. During these measurements, the low power probe beam did not exceed $0.3 \mathrm{~W}$. This corresponds to an intensity of $9.5 \mathrm{~W} /$ $\mathrm{cm}^{2}$, which is well below the reported saturation intensity of $45-189 \mathrm{~W} / \mathrm{cm}^{2} .{ }^{13,23}$ A gas flow rate of $\sim 1 \mathrm{SCHF}$ was used during all gain measurements.

Due to discharge instability and arcing at lower ratios of $\mathrm{He} / \mathrm{CO}_{2}$, it was difficult to examine the gain 


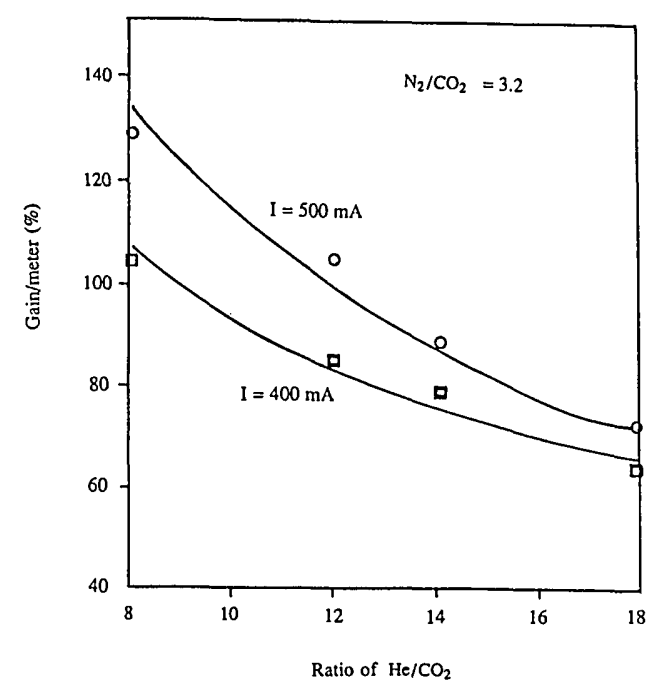

Fig. 5. Gain as a function of $\mathrm{He} / \mathrm{CO}_{2}$ ratio at 7 Torr.

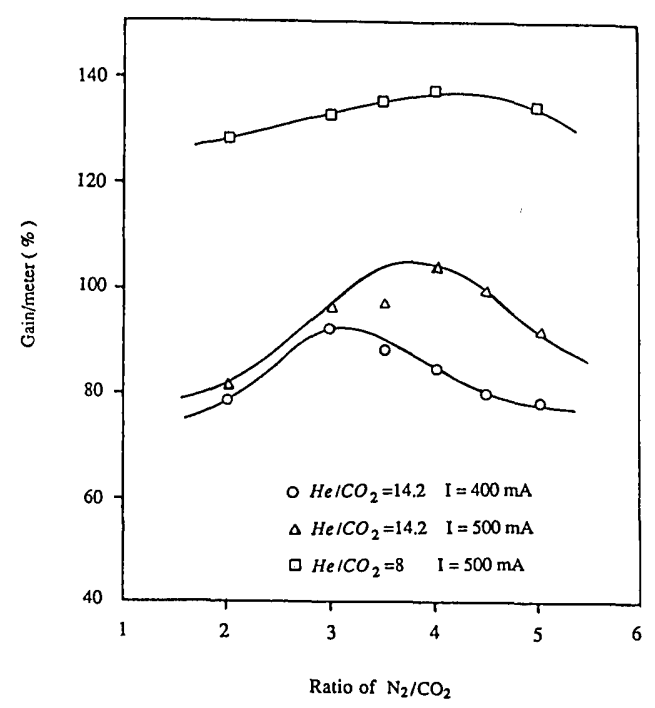

Fig. 6. Gain as a function of $\mathrm{N}_{2} / \mathrm{CO}_{2}$ ratio at 7 Torr.

when the ratio of $\mathrm{He} / \mathrm{CO}_{2}$ is $<8$. The optimum choice of gas mixture to have a stable discharge and a relatively high gain was at a $\mathrm{CO}_{2}: \mathrm{N}_{2}: \mathrm{He}$ ratio of $\sim 1: 3.2: 14.2$. From Figs. 5 and 6, the highest measured gain is $\sim 140 \% / \mathrm{m}$ for a mixture of $1: 3.2: 8$ at a current of $0.5 \mathrm{~A}$. The gain increases as the ratio of $\mathrm{He} / \mathrm{CO}_{2}$ decreases, and the gain is higher at higher current as shown in Fig. 5. From Fig. 6, the gain is highest when the ratio of $\mathrm{N}_{2} /$ $\mathrm{CO}_{2}$ is 2-3.5. The sensitivity of the gain to the $\mathrm{N}_{2} / \mathrm{CO}_{2}$ ratio increases as the $\mathrm{He} / \mathrm{CO}_{2}$ ratio increases.

The effect of pressure on gain for two gas mixtures is shown in Fig. 7. Gas mixtures with lower $\mathrm{He} / \mathrm{CO}_{2}$ ratios were not considered here due to arcing and discharge instability at higher pressure. Figure 7 illustrates that the gain increases with increasing pressure until it reaches a maximum and then decreases due to increases in temperature. ${ }^{13}$ At higher pressure, the discharge contracts to the cathode surface.

The transverse gain profiles across the waveguide (cathode) were measured for different gas mixtures at

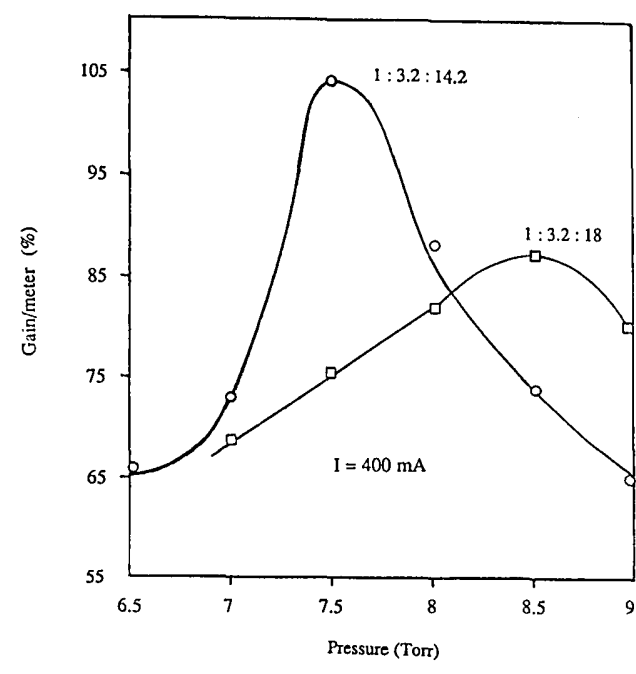

Fig. 7. Gain as a function of pressure.

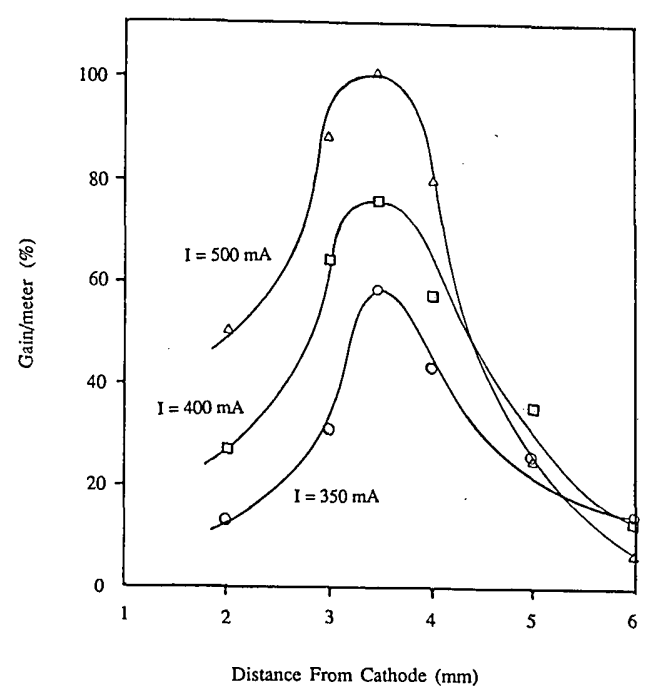

Fig. 8. Gain profile for the 1:3.2:14.2 gas mixture at 7 Torr.

different discharge currents and pressures. Figure 8 shows the results of some of these measurements. This figure and other measurements reveal that close to the cathode at the end of the intense part of the negative glow the gain increases with increasing distance from the cathode until a maximum value is reached. Beyond this point, the gain decreases. The peaks of the gain curves increase and shift toward the cathode surface with increasing pressure and with lower ratios of $\mathrm{He} / \mathrm{CO}_{2}$. The gain curves also are flattened somewhat with higher ratios of $\mathrm{He} / \mathrm{CO}_{2}$. This is important, since more uniform gain may lead to better laser mode quality. ${ }^{23}$ These measurements also reveal that an increase in the ratio of $\mathrm{He} / \mathrm{CO}_{2}$ beyond eight causes an increase in the intense part of the negative glow, and this shifts the gain region away from the cathode surface while reducing the peak value of the gain. In general, the gain peaks tend to be located between 2 and $4 \mathrm{~mm}$ from the surface of the cathode.

Due to arcing at higher currents, the range of cur- 
rents was kept between 300 and $500 \mathrm{~mA}$. The gain is found to increase with current in this range, except for very high He levels, and it is found, for example, that for the ratio of 1:3.2:18 the gain is lower at $500 \mathrm{~mA}$ than at $400 \mathrm{~mA}$. This may be due to an observable increase in length of the intense part of the negative glow with current. Also, for a given $E / N$ the average electron energy increases when the proportion of $\mathrm{He}$ is increased, because $\mathrm{He}$ has none of the inelastic vibrational losses that are present in $\mathrm{CO}_{2}$ and $\mathrm{N}_{2}$. Thus, electron energies sufficient to excite the upper laser level occur at lower $E / N .^{25}$

The previous measurements illustrate the availability of high gain in this device and show that the optimum choice for gas mixture ratio and gas pressure is $\sim$ 1:3.2:14.2 of $\mathrm{CO}_{2}: \mathrm{N}_{2}: \mathrm{He}$ and 7 Torr, respectively.

\section{Metal Waveguide Laser}

The amplifier studied in the last section is turned into a laser medium by placing it inside an optical resonator. This optical resonator consists of two mirrors, one is a $1-\mathrm{m}$ curvature gold coated mirror and the other is a flat germanium mirror with $95 \%$ reflectivity. The waveguide length is $42 \mathrm{~cm}$, and the distance between each mirror and the end of the waveguide is $\sim 3-$ $5 \mathrm{~mm}$. The mirror spacing from the waveguide, which controls the coupling efficiency between the modes of the waveguide and the modes of free space, has been studied by Abrams and Chester. ${ }^{26}$

The modes of curved concave metal waveguides have been investigated theoretically and experimentally by Casperson and Garfield ${ }^{8}$ and Marhic et al..$^{11,27}$ They showed that these waveguide modes could be described in terms of Hermite-Gaussian functions parallel to the waveguide surface and Airy functions in the perpendicular direction. The results of their study are briefly summarized, and the coordinate system shown in Fig. 9 will be used in this discussion. The major and minor radii of curvature are $r_{0} \approx 46 \mathrm{~m}$ and $R_{0}$ $=5.5 \mathrm{~mm}$, respectively, $r^{\prime}$ is the distance perpendicular to the waveguide surface, $z$ is the distance across the waveguide, and $\phi$ is the angular distance along the waveguide. by $^{8}$

Using this system of units the electric field is given

$$
\begin{aligned}
E_{m n}\left(r^{\prime}, \phi, z\right)= & E_{0} F\left[\left(2 \frac{k_{0}^{2}}{r_{0}}\right)^{\frac{1}{3}} r^{\prime}-\rho_{n}\right] H_{m}\left(\frac{\sqrt{2} z}{w_{s}}\right) \\
& \times \exp \left(\frac{z^{2}}{w_{s}^{2}}\right) \exp \left[i\left(\psi-k_{0} z\right)\right],
\end{aligned}
$$

where $E_{0}$ is the normalizing factor, $\rho_{n}$ is the $n$th zero of the Airy function $F, k_{0}$ is the free space propagation constant, $H_{m}$ is the Hermite-Gaussian function, $\psi$ is a phase shift, and $w_{s}$ is the steady state spot size given by

$$
w_{s}=\left[\frac{2\left(r_{0} R_{0}\right)^{1 / 2}}{k_{0}}\right]^{1 / 2} \text {. }
$$

If we were to neglect the effect of the waveguide walls on the propagating wave, the modes would be free

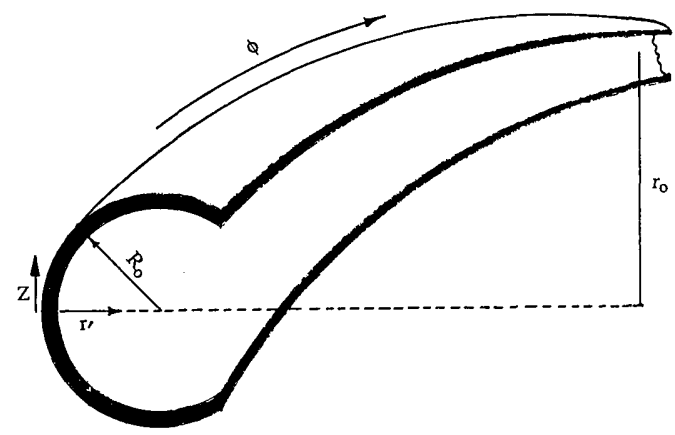

Fig. 9. Waveguide coordinate system.

space modes. The Cartesian free space electric field modes for waves propagating in the $z$ direction are given by

$$
\begin{aligned}
E_{m n}(x, y, z)= & E_{0} \frac{w_{0}}{w(z)} H_{m}\left(\frac{\sqrt{2} x}{w(z)}\right) H_{n}\left(\frac{\sqrt{2} y}{w(z)}\right) \\
& \times \exp \left[-i k_{0} \frac{x^{2}+y^{2}}{2 R(z)}-\frac{x^{2}+y^{2}}{w^{2}(z)}\right] \exp \left[i\left(k_{0} z+\psi\right)\right],
\end{aligned}
$$

where $R(z)$ is the radius of curvature and $w(z)$ is the spot size of the beam at a distance $z$ from the waist $w_{0}$. The spot size is given by

$$
w^{2}(z)=w_{0}^{2}\left[1+\left(\frac{z}{z_{0}}\right)^{2}\right]
$$

for a free space mode. In our half-symmetric resonator the waist spot size would be

$$
\begin{aligned}
& w_{0}^{2}=\frac{\lambda}{\pi}(d R)^{1 / 2}\left(1-\frac{d}{R}\right)^{1 / 2}, \\
& z_{0}=\frac{\pi w_{0}^{2}}{\lambda},
\end{aligned}
$$

where $\lambda$ is the wavelength, $d$ is the cavity length, and $R$ is the radius of curvature of the curved mirror.

The laser beam is IR, and its cross section has been made visible by letting the beam strike a fluorescing surface of a thermal image plate. The output modes were always nearly perfect lower-order modes. This suggests that the gain in the middle of the waveguide along the optical axis is highest and decreases sharply away from it. Also, the cathode-anode separation acts as an aperture.

At low current and pressure of $600 \mathrm{~mA}$ and 7 Torr, respectively, the optimum output modes were freespace lower-order Hermite-Gaussian modes. At higher current and pressure, $900 \mathrm{~mA}$ and 10 Torr, respectively, the optimum modes were low-order AiryHermite-Gaussian modes. This difference in mode shapes can be explained as follows: at low current and pressure, the thickness of the gain medium is smaller than the distance between the gain medium and waveguide surface. This makes it almost impossible to make the beam use the waveguide surface as a guiding medium because the medium between the gain medium and the waveguide walls is thick and lossy. But at high current and pressure, the gain is high very close to the waveguide surface. Therefore, the system lases best when the mirrors are tilted causing the beam to 


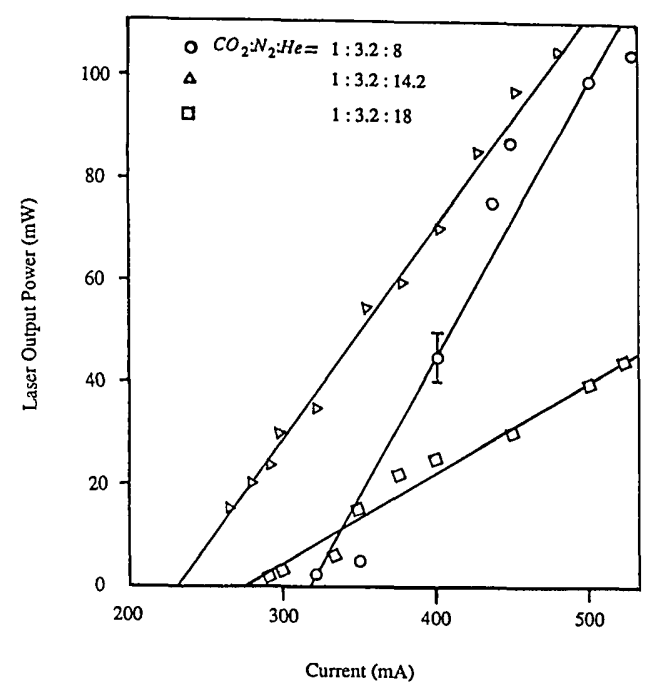

Fig. 10. Laser power as a function of current for different gas mixtures at 7 Torr.

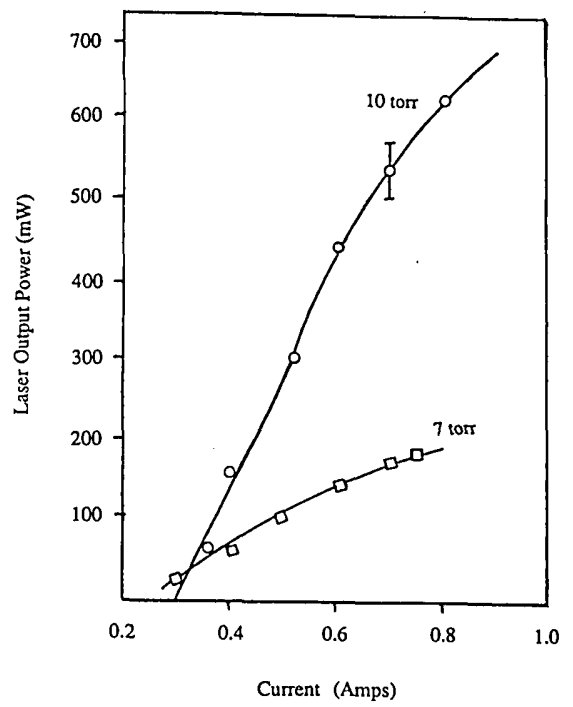

Fig. 11. Laser power as a function of current for the 1:3.2:14.2 gas mixture at 7 and 10 Torr.

follow the waveguide surface. In these conditions we get the waveguide modes.

One of the most important aspects of a laser's performance is the power dependence on current. We have measured the laser's output power as a function of current for different gas mixtures and pressures. These measurements were done using a Scientech model 362 power meter.

Figure 10 shows that at 7 Torr the power is almost linearly proportional to the current up to over $500 \mathrm{~mA}$ for the different gas mixtures shown. But for higher current, Fig. 11 illustrates that the power increases less with current. The reasons for this behavior may be due to increases in temperature and a faster rate of gas contamination. Finally, it may be observed that for the same gas mixture ratio, flow rate, and energy input, the efficiency of the waveguide laser is the same or better than that of the conventional longitudinal laser used in our gain measurements. The maximum $\mathrm{TEM}_{00}$ power obtained from this metal waveguide laser was over $1 \mathrm{~W}$.

\section{Conclusion}

The design and operation of a dc-excited cw $\mathrm{CO}_{2}$ metal waveguide laser, in which a slotted hollow cathode in a transverse discharge also doubles as a metal waveguide has been presented. The resulting transverse discharge has many advantages which include low voltage excitation, positive impedance, discharge homogeneity along the optical axis, and the unexpected high gain of the special Faraday dark space formed near the center of the cathode. Another attractive feature is the high thermal conductivity of the cathode walls, which leads to increased optical gain. The discharge was found to be stable at ratios of $\mathrm{He} / \mathrm{CO}_{2}$ higher than 8, and the gain was found to increase with current and pressure.

Lasing has been achieved using the above amplifier. The output transverse modes are found to be clean lower-order modes due to the uniformity and shape of the gain medium which favor such modes. The modes were found to be either free space modes or waveguide modes depending on the discharge conditions. The lower currents and pressures tend to favor the Hermite-Gaussian free space modes, while the higher currents and pressures favor the Airy-Hermite-Gaussian waveguide modes. A maximum $\mathrm{TEM}_{00}$ power output of over $1 \mathrm{~W}$ has been obtained.

This work was supported in part by the National Science Foundation under grant ECS-8511593 and by Tektronix, Inc.

\section{References}

1. P. W. Smith, "A Waveguide Gas Laser," Appl. Phys. Lett. 19, 132-134 (1971).

2. J.J.Degnan, “The Waveguide Laser: A review," Appl. Phys. 11, 1-33 (1976).

3. P. W. Smith, O. R. Wood, P. J. Maloney, and C. R. Adams, "Transversely Exited Waveguide Gas Lasers," IEEE J. Quantum Electron. QE-17, 1166-1181 (1981).

4. E. A. J. Marcatili and R. A. Schmeltzer, "Hollow Metallic and Dielectric Waveguides for Long Distance Optical Transmission and Lasers," Bell Syst. Tech. J. 43, 1783-1809 (1964).

5. H. Krammer, "Field Configuration and Propagation Constants of Modes in Hollow Rectangular Dielectric Waveguides," IEEE J. Quantum Electron. QE-12, 505-507 (1976).

6. B. Adam and F. Kneubuhl, "Transversely-Excited $337 \mu \mathrm{m} \mathrm{HCN}$ Waveguide Laser," Appl. Phys. 8, 281-291 (1975).

7. E. Garmire, T. McMahon, and M. Bass, "Propagation of Infrared Light in Flexible Hollow Waveguides," Appl. Opt. 15, 145150 (1976).

8. L. W. Casperson and T. S. Garfield, "Guided Beams in Concave Metallic Waveguides," IEEE J. Quantum Electron. QE-15, 491496 (1979).

9. D. A. Pinnow et al., "Polycrystalline Fiber Optical Waveguides for Infrared Transmission," Appl. Phys. Lett. 33, 28-29 (1978).

10. Y. Mimura and C. Ota, "Transmission of $\mathrm{CO}_{2}$ Laser Power by Single-Crystal CsBr Fibers," Appl. Phys. Lett. 40, 773-775 (1982).

11. M. E. Marhic, L. I. Kwan, and M. Epstein, "Whispering-Gallery 
$\mathrm{CO}_{2}$ Laser," IEEE J. Quantum Electron. QE-15, 487-490 (1979).

12. J. G. Grossman, L. W. Casperson, and O. M. Stafsudd, "RadioFrequency-Excited Carbon Dioxide Metal Waveguide Laser," Appl. Opt. 22, 1298-1305 (1983).

13. K. T.-K. Cheng and L. W. Casperson, "Properties of a Coaxial cw $\mathrm{CO}_{2}$ Laser," Appl. Opt. 18, 2130-2135 (1979).

14. D. J. Sturges and H. J. Oskam, "Hollow-Cathode Discharge in Hydrogen and Noble Gases," J. Appl. Phys. 37, 2405-2412 (1986).

15. R. F. Little and A. V. Engel, "The Hollow-Cathode Effect and Theory of Glow Discharge," Proc. R. Soc. London Ser. A 224, 209-227 (1954).

16. K. Fujii et al., "Design of Whitelight Laser Based on Cathode Fall Theory," IEEE J. Quantum Electron. QE-15, 35-43 (1979).

17. S. Hashiguchi and M. Hasikuni, "Theory of the Hollow Cathode Glow Discharge,” Jpn. J. Appl. Phys. 26, 271-280 (1987).

18. V. P. Chebotayev, "Operating Condition of an Optical Maser Containing a He-Ne Mixture," Radio Eng. Electron. Phys. USA 10, 314-316 (1965).

19. S. C. Wang and A. E. Siegman, "Hollow-Cathode Transverse Discharge He-Cd+ Lasers," Appl. Phys. 2, 143-150 (1973).
NASA continued from page 1812

then have multiple opportunities to photoexcite holes in the thin metal silicide layers. To achieve an overall quantum efficiency of $\sim 10 \%$ or better in a multiple layer device, the metal silicide layers must be thinner than $\sim 10 \mathrm{~nm}$; that is, substantially less than a meanfree-path length for the photoexcited holes. This ensures that the holes have repeated opportunities of being collected at one of the interfaces before losing their energy by inelastic collisions.

Detectors of the new type could be grown by molecular beam epitaxy on silicon wafers that also contain very-large-scale integrated circuits. Thus, imaging arrays of detectors could be made in monolithic units with image preprocessing circuitry.
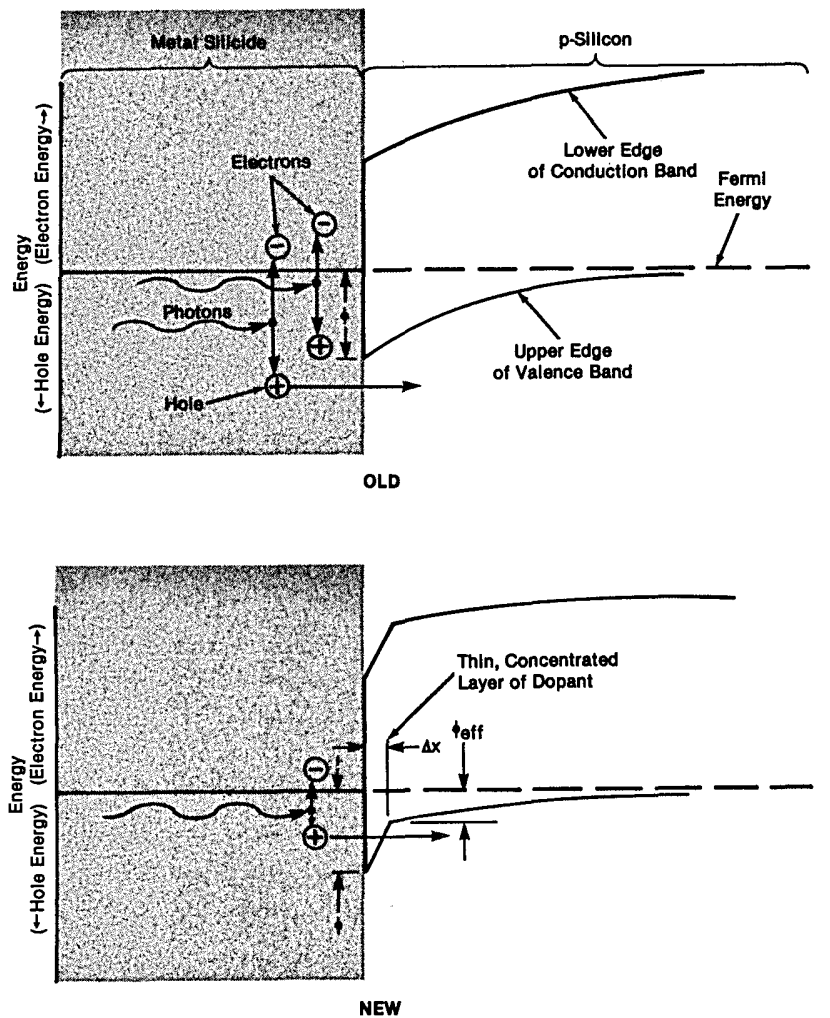

Fig. 4. Thin concentrated layer of dopant would narrow the Schottky barrier, reducing its effective height from $\phi$ to $\phi_{\text {eff. }}$.
20. C. S. Willet and G. M. Janny, "Amplification at $10.6 \mu$ in the Negative Glow of Hollow Cathode Discharge in a $\mathrm{CO}_{2}-\mathrm{He} \mathrm{Mix}-$ ture," IEEE J. Quantum Electron. QE-6, 568-569 (1970).

21. R. J. Frieberg and P. O. Clark, " $\mathrm{CO}_{2}$ Transverse Discharge Lasers," IEEE J. Quantum Electron. QE-7, 581-583 (1971).

22. E. T. Gerry and D. A. Leonard, "Measurement of 10.6 microns $\mathrm{CO}_{2}$ Laser Transition Probability and Optical Broadening Cross Section," Appl. Phys. Lett. 8, 227-229 (1966).

23. V.A. Seguin et al., "Gain Characteristics of a MAGPIE Coaxial $\mathrm{CO}_{2}$ Laser System," IEEE J. Quantum Electron. QE-23, 600604 (1987).

24. A. E. Siegman, An Introduction to Lasers and Masers (McGraw-Hill, New York, 1968), Chap. 3.

25. J. J. Lowke, A. V. Phelps, and B. W. Irwin, "Predicted Electron Transport Coefficients and Operating Characteristics of $\mathrm{CO}_{2-}$ $\mathrm{N}_{2}$-He Laser Mixture,” J. Appl. Phys. 44, 4664-4471 (1973).

26. R. L. Abrams and A. N. Chester, "Resonator Theory for Hollow Waveguide Lasers," Appl. Opt. 13, 2117-2125 (1974).

27. M. E. Marhic, L. I. Kwan, and M. Epstein, "Optical Surface Waves Along a Toroidal Metallic Guide," Appl. Phys. Lett. 33, 609-611 (1979).

This work was done by Joseph Maserjian of Caltech for NASA's Jet Propulsion Laboratory. Inquiries concerning rights for the commercial use of this invention should be addressed to the patent counsel, P.F. McCaul, NASA Resident Office-JPL, Mail Code 180801, 4800 Oak Grove Drive, Pasadena, CA 91109. Refer to NPO17194.

\section{Optical sensor of high gas temperature}

An optical fiber temperature sensor withstands hot engine exhaust gases. The sensor is more reliable than thermocouples or resistance temperature devices, the wires of which are subject to heat- and vibration-induced failure. It is more accurate than conventional pyrometers, which are prone to errors caused by shifts in the emissivities of gases with changes in temperature and composition.

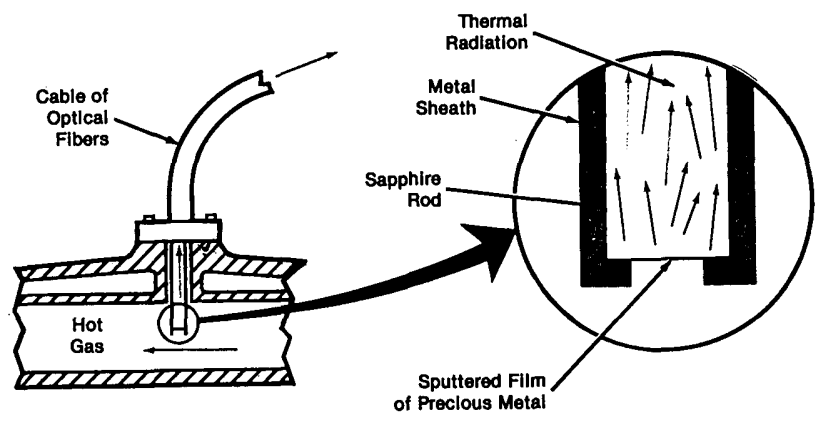

Fig. 5. Optical fiber sensor projects into a stream of hot gas. The temperature of the metal film on the tip of the sensor is essentially that of the gas. An optical fiber cable conducts thermal radiation from the film to a photodetector.

The new sensor consists of a shielded sapphire rod with a sputtered layer of precious metal on its end (see Fig. 5). The metal layer acts as a blackbody. It emits radiation that has a known dependence of spectral distribution with the temperature of the metal and, consequently, with the temperature of the hot gas flowing over the metal. A fiber optic cable carries the radiation from the sapphire rod to a remote photodetector.

This work was done by Arthur J. Hill of Rockwell International Corp. for Marshall Space Flight Center. Refer to MFS-29316.

continued on page 1909 